\title{
Electron backscatter diffraction study of orientation gradients at the grain boundaries of a polycrystalline steel sheet deformed along different loading paths
}

\author{
J. W. Signorelli, A. Roatta, N. De Vincentis, C. Schwindt, M. Avalos, R. E. \\ Bolmaro and N. Bozzolo
}

J. Appl. Cryst. (2017). 50, 1179-1191

\section{- IUCr Journals CRYSTALLOGRAPHY JOURNALS ONLINE}

Copyright (C) International Union of Crystallography

Author(s) of this paper may load this reprint on their own web site or institutional repository provided that this cover page is retained. Republication of this article or its storage in electronic databases other than as specified above is not permitted without prior permission in writing from the IUCr.

For further information see http://journals.iucr.org/services/authorrights.html 
JOURNAL OF

APPLIED

CRYSTALLOGRAPHY

ISSN 1600-5767

Received 30 November 2016

Accepted 23 June 2017

Edited by A. Borbély, Ecole National Supérieure des Mines, Saint-Etienne, France

Keywords: orientation gradients; electron backscatter diffraction; EBSD; plastic deformation; steel sheets; in-plane loading conditions.

Supporting information: this article has supporting information at journals.iucr.org/j

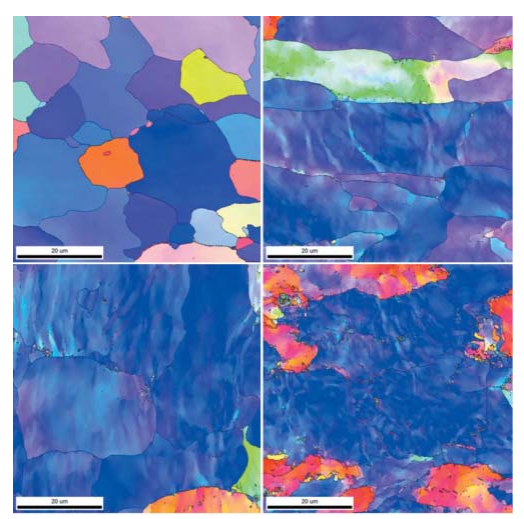

C 2017 International Union of Crystallography

\section{Electron backscatter diffraction study of orientation gradients at the grain boundaries of a polycrystalline steel sheet deformed along different loading paths}

\author{
J. W. Signorelli, ${ }^{a, b *}$ A. Roatta, ${ }^{a, b}$ N. De Vincentis, ${ }^{a}$ C. Schwindt, ${ }^{c}$ M. Avalos, ${ }^{a, b}$ R. E. \\ Bolmaro $^{a, b}$ and N. Bozzolo ${ }^{d}$
}

${ }^{\mathbf{a}}$ IFIR - UNR - CONICET, Bulevar 27 de Febrero 210b, Rosario (S2000BTP), Santa Fe, Argentina, ${ }^{\mathbf{b}}$ Facultad de Ciencias Exactas, Ingeniería y Agrimensura (FCEIA-UNR), Avenida Pellegrini 250, Rosario, 2000, Argentina, 'Department of Engineering, UNS-CONICET, Avenida Alem 1253, Bahía Blanca, Buenos Aires, B8000CPB, Argentina, and ${ }^{\mathbf{d} M I N E S}$ ParisTech, PSL - Research University, CEMEF, CNRS UMR 7635 CS 10207, 1 rue Claude Daunesse, Sophia Antipolis Cedex, 06904, France. *Correspondence e-mail: signorelli@ifir-conicet.gov.ar

In a polycrystal, the heterogeneity of plastic deformation in a particular grain is greatly enhanced by adjacent grains that constrain the grain's local behavior, often imposing orientation gradients. This work aims to characterize and quantify the local orientation gradients near grain boundaries (GBs). Electron backscatter diffraction (EBSD) measurements were made on a $0.67 \mathrm{~mm}$ thick aluminium-killed drawing quality (AKDQ) steel sheet subjected to different loading paths that are typical of forming operations. A statistical analysis shows that a considerable fraction of the analyzed GB profiles can be described by an orientation profile with a constant slope near the GB. In order to quantify this behavior, as well as the degree of localization, two new parameters, based on the local orientation gradient assessed by EBSD, are proposed: BET (boundary effective thickness) and GAS (gradient average severity). These parameters should be considered together, the BET as an effective thickness of the GB zone where the orientation gradient takes place and the GAS as a measure of the magnitude or severity of the orientation gradient. Additionally, the GAS parameter shows a strong correlation with the accumulated macroscopic strain for the investigated deformation levels and loading paths, while the BET profile clearly reveals the influence of the GB on the misorientation profiles. Tension and biaxial stretching results lead to a BET value between 1.5 and $2 \mu \mathrm{m}$. Finally, it is shown that the local misorientation in the GB zone, on both sides of the GB line, is disperse and it does not correlate simply with misorientation or even the slip-transfer geometry across the GB. Moreover, the observed average local misorientation dispersions in GB zones are different for each loading condition.

\section{Introduction}

Stretching and deep-drawing processes have been used for many years to form parts from a variety of sheet metals. These forming operations involve plastic deformation, which is, by nature, inhomogeneous. As deformation proceeds, the accumulation of dislocations inside grains produces local crystallattice rotations (i.e. local misorientations), and low- followed by high-angle boundaries, which can all be quantified as local orientation gradients. Experimental and numerical research for a variety of polycrystalline materials has shown that these gradients result from a complex phenomenon involving the combined effects of loading conditions and the microscale behavior of the grains constituting the polycrystalline aggregate. Several factors contribute to the character of local 
deformation, such as the topology and the shape of the crystals, the grain boundary spatial distribution, and the individual crystal orientation (Kanjarla et al., 2010; Rollett et al., 2012; Franciosi \& Berbenni, 2008; Allain-Bonasso et al., 2012; Scheriau \& Pippan, 2008; Mishra et al., 2009; Lebensohn et al., 2008; Winther, 2008).

Mishra et al. (2009) characterized orientation gradients between the grain interior and the grain boundary in terms of the length over which a significant gradient was observed and the maximum misorientation angle, in aluminium samples deformed in uniaxial tension. They observed that the estimated length ratios correlate with differences in the yield behavior of the grain pair (an inverse power-law relationship with the Taylor factor ratio). These results offer evidence of a near-neighbor grain interaction. Rollett et al. (2012) dealt with orientation gradients near grain boundaries in an interstitialfree steel sheet and found neither a relationship between orientation gradient and grain size nor a correlation with grain orientation. On the other hand, Winther (2008) showed that both lattice rotations and dislocation structures depend on the grain orientation in aluminium polycrystals deformed by tension or rolling. Using orientation imaging microscopy and full field simulations, Lebensohn et al. (2008) studied the orientation dependence of the average misorientations in polycrystalline $\mathrm{Cu}$ deformed in tension. They showed that the interactions with different neighbors may be responsible for the local rotation towards two different stable orientations. This leads to the formation of subdomains inside grains and the consequent development of intragranular misorientations. Orientation gradients are also found in ferrite-martensite microstructures, like in dual-phase steels. In two ultrafinegrained dual-phase steels with different martensite island sizes and volume fractions, Calcagnotto et al. (2010) quantified orientation gradients, pattern quality and density of geometrically necessary dislocations (GNDs) at ferrite-ferrite and ferrite-martensite interfaces. They found that even very small martensite islands cause strong local plastic deformation in the adjacent ferrite regions and important orientation gradients close to the ferrite-martensite grain boundaries (GBs). In a $\mathrm{Cu}$ polycrystal, with grain sizes ranging between 5 and $50 \mu \mathrm{m}$, Soifer et al. (2002) observed heterogeneous plastic deformation near grain boundaries. They found that the hardness near the GB increases by a factor of 1.5 compared to the core of the grain. More important, the effect of the GB was significant as far away as $2 \mu \mathrm{m}$. Soer et al. (2005) studied the near-GB hardening in Mo bicrystals and established that the GB affects the hardening over a $1 \mu \mathrm{m}$ wide zone.

Moreover, orientation gradients play an important role in grain fragmentation, in recrystallization and in the development of stress concentrations near GBs, which may eventually lead to crack initiation (Takayama et al., 2005; Bieler et al., 2009; Rollett et al., 2007; Pouillier et al., 2012). Recently, researchers have studied the localization of plastic strain associated with heterogeneous deformation near GBs, as a precursor to the nucleation of fatigue cracks. This was done to correlate the influence of the geometrical configuration of slip on both sides of the grain boundary. Abuzaid et al. (2012) provided pointwise comparisons between strain fields and microstructure and correlated the plastic strain magnitudes across the GBs with the residual Burgers' vector. Bieler et al. (2014) showed that slip transfer can be rationalized in terms of a geometrical alignment of activated slip systems in neighboring grains.

Nowadays, electron backscatter diffraction (EBSD) allows accurate maps of crystal orientations to be easily obtained. Several EBSD-derived parameters can be found in the literature, reflecting the heterogeneity of plastic deformation inside grains (Jorge-Badiola et al., 2007; Wagner et al., 2012; Allain-Bonasso et al., 2012; Githinji et al., 2013; Schayes et al., 2016). Among these, the kernel average misorientation (KAM) is noteworthy (Subedi et al., 2015; Rollett et al., 2012). The KAM can be used as a simple metric to quantify a GND density compatible with the orientation field (Moussa et al., 2015; Wright et al., 2015). The KAM parameter greatly depends on measurement conditions (sample preparation procedure and surface quality, pattern resolution, and resolution of the Hough space within which the position of the Kikuchi bands is detected) as well as the size ratio between the investigated structures and the chosen step size (Kamaya, 2011). Allain-Bonasso et al. (2012) discussed the use of several of these EBSD parameters for the characterization of plastic heterogeneities. They showed that the grain orientation spread (GOS) and moreover GOS/D (GOS divided by the grain diameter, $D$ ) allow recognition of grains that have undergone high or low deformation. More recently, Subedi et al. (2015) studied the relationship between orientation gradients and grain boundaries, by varying the strain level and the scan step, in a high-purity polycrystalline copper pulled to failure in tension. They paid particular attention to the scanstep effect on the KAM calculation. In their case, a scan step of $0.5 \mu \mathrm{m}$ was sufficiently fine to sample the dislocation substructure with enough resolution to capture all the postdeformation features. Kamaya $(2011,2012)$ investigated, also by EBSD, the localization of misorientation near grain boundaries and the distribution of local misorientations, in fatigued stainless-steel specimens. He showed that the local misorientation tended to concentrate at GBs and the intensity of the localization increased with the applied deformation. Additionally, he reported poor correlations between the local misorientation near a grain boundary $\left(M_{\mathrm{GB}}\right)$ and the $\mathrm{GB}$ length, or between $M_{\mathrm{GB}}$ and the misorientation across the GBs, including twin boundaries.

The present study focuses on an aluminium-killed drawing quality (AKDQ) steel sheet. This material is produced especially for making parts that require severe forming or drawing operations, under conditions that are beyond the limits of commercial quality cold-rolled steel sheets. AKDQ steel is often taken as a reference because of its many applications and production volume. The steel's drawability, ductility and surface appearance are of great importance in most of its uses, including a variety of applications ranging from automotive bodies to appliances and cans. Improvements in properties can be achieved by suitable thermomechanical treatment. Accordingly, a detailed study of the manufacturing parameters, 
the mechanical properties, the final microstructures and their interdependent relations is needed to optimize processing parameters and forming paths.

This work aims at obtaining relevant quantitative information from EBSD maps to characterize plastic strain heterogeneities and orientation gradients in AKDQ steel sheet samples subjected to different deformation conditions near necking. The paper is organized as follows. The experimental section presents the material characterization and mechanical tests. Then, the basic definitions related to crystal misorientation and parameters representing the localization of crystal misorientations are reviewed. The validity of assessing mesoscopic deformation from EBSD parameters is discussed, and subsequently, orientation gradients within grain boundary zones (GBZs) are analyzed to determine what fraction of them can be statistically quantified into a simple profile. To further characterize this behavior, two new EBSD-derived parameters are proposed. These parameters account for the magnitude or severity of the orientation gradient and the effective width along the boundary where it occurs. Finally, the correlation between the geometry of slip transfer across the $\mathrm{GB}$ and the loading path is considered.

\section{Experimental procedure}

A $0.67 \mathrm{~mm}$ thick electrogalvanized AKDQ steel sheet with an ASTM grain size number of 9-10 (ASTM E112) and Vickers' hardness of $106 \mathrm{HV}_{5}$ was used in this investigation. The chemical composition of the steel, excluding the zinc plating, is given in Table 1.

Nakajima samples were laser cut and tested in three stages (Signorelli et al., 2012): application of a grid of circles to the samples; punch stretching to maximum load; and measurement of strains. The grid pattern of non-contacting $2.50 \mathrm{~mm}$ diameter circles was printed on the sheet samples by electrochemical deposition. The specimen was then mounted with its gridded side opposite a $40 \mathrm{~mm}$ diameter hemispherical punch and stretched with a punch velocity of $0.5 \mathrm{~mm} \mathrm{~min} \mathrm{~m}^{-1}$ up to a noticeable drop in the load, indicating the presence of localized necking. The punch and die setup is shown in Fig. 1. Multiple strain states were obtained by varying the width of the hourglass-shape samples and using adequate lubrication conditions. After testing, the major and minor dimensions of the ellipses, with both uniform and localized deformations, were measured with a profile projector. The hourglass geometries were designed to approximate uniaxial tension (UAT), plane strain (PS) and equibiaxial (EBA) stretching conditions (Fig. 1).

The as-received (AR) material texture was measured using X-ray diffraction in a Phillips X'Pert Pro MPD system equipped with a texture goniometer, a $\mathrm{Cu} K \alpha$ radiation source and polycapillary optics. The AR texture exhibits the typical characteristics of cold-rolled and annealed steels. Microstructure characterization revealed the presence of two types of grains. Some grains have a spherical shape associated with
Table 1

Chemical composition of the AKDQ steel sheet.

\begin{tabular}{lllllllllll}
\hline Element $\mathrm{C}$ & $\mathrm{P}$ & $\mathrm{S}$ & $\mathrm{Mn}$ & $\mathrm{Si}$ & $\mathrm{Cr}$ & $\mathrm{Ni}$ & $\mathrm{Cu}$ & $\mathrm{Mo}$ & $\mathrm{Al}$ & (p.p.m.)
\end{tabular}

$\begin{array}{llllllllllll}\mathrm{W} t \% & 0.070 & 0.020 & 0.009 & 0.300 & 0.040 & 0.020 & 0.010 & 0.010 & 0.010 & 0.19288\end{array}$

partial recrystallization, while others are slightly elongated in the rolling direction (Signorelli et al., 2012).

For each sample, a $3 \times 5 \mathrm{~mm}$ area close to the necking zone, but not including the area of plastic instability, was selected in order to analyze the development of orientation gradients for each deformed state. After cutting, each sample was prepared for EBSD measurements. The surface was mechanically polished with 1200 grit paper, followed by $9,6,3$ and $1 \mu \mathrm{m}$ diamond pastes and $0.05 \mu \mathrm{m}$ colloidal silica. Sample finishing with colloidal silica assures a flat scratch-free surface, which is required for EBSD microstructural analysis. Local orientations were measured using an FEI QUANTA 200 scanning electron microscope, equipped with a field emission gun and

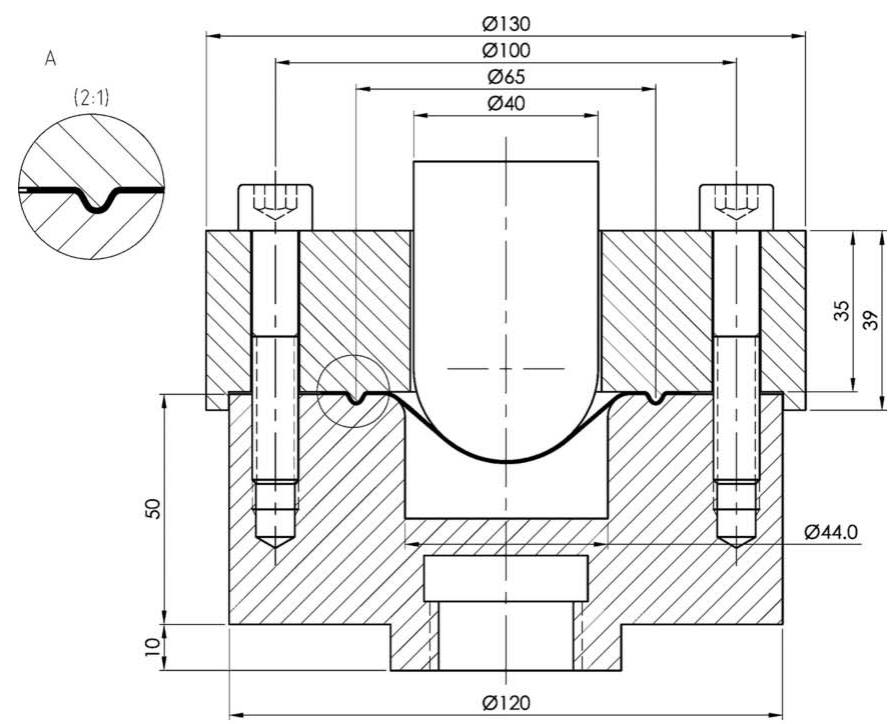

(a)

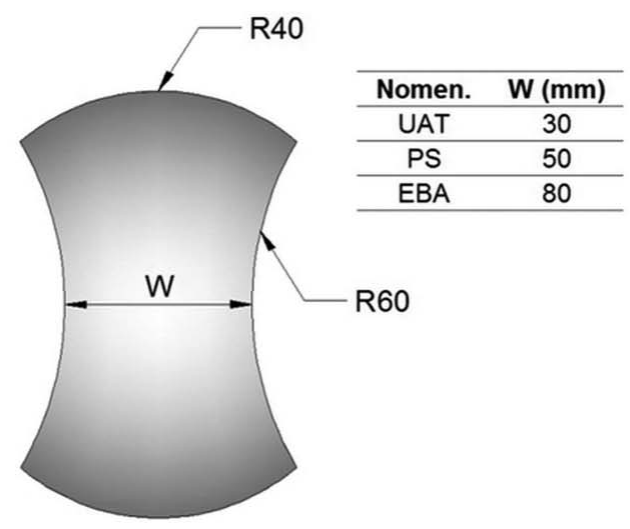

(b)

Figure 1

Details of $(a)$ the punch and die setup and $(b)$ the dimensions of the sheet samples used to obtain different strain states. 
Table 2

Summary of the performed EBSD measurements.

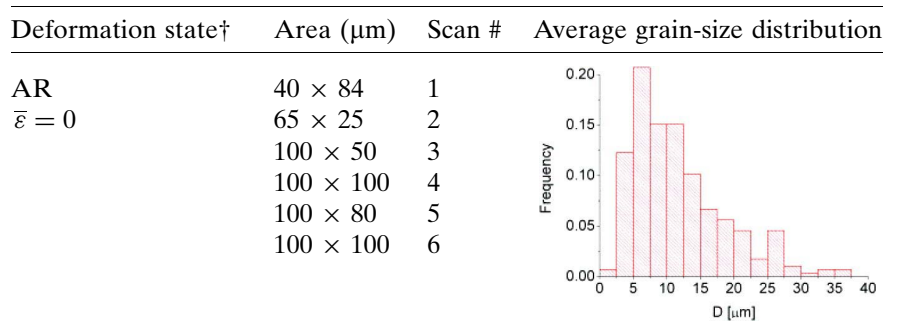

$\begin{array}{lll}\text { UAT } & 100 \times 80 & 7 \\ \bar{\varepsilon}=0.44 ; \rho=-0.23 & 110 \times 70 & 8 \\ & 62 \times 75 & 9 \\ & 55 \times 50 & 10 \\ & 120 \times 110 & 11 \\ & 100 \times 100 & 12 \\ & & \\ & \\ & \\ \text { PS } & \\ \bar{\varepsilon}=0.39 ; \rho=0.13 & 65 \times 60 & \\ & 65 \times 60 & 13 \\ & 65 \times 60 & 15 \\ & 100 \times 100 & 16 \\ & 100 \times 100 & 17\end{array}$
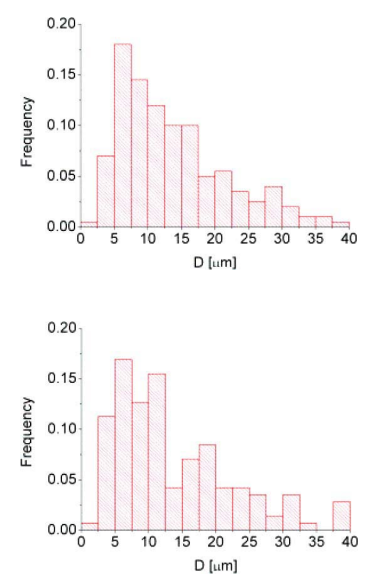

EBA

$65 \times 65 \quad 19$

$65 \times 65 \quad 20$

$100 \times 45 \quad 21$

$100 \times 45 \quad 22$

\section{Results and discussion}

\subsection{Assessment of local deformation}

Inherent effects from the influence of step size on the accuracy of local misorientation data complicate the extraction of relevant information from EBSD maps (Kamaya, 2011; Moussa et al., 2015; Wright et al., 2015). Assuming a local linear correlation between the misorientation angle and the kernel size (i.e. a constant orientation gradient at the kernel scale), Kamaya (2011) proposed two parameters, the local gradient of crystal orientation, $G_{\mathrm{L}}$, and the background noise, $B_{\mathrm{L}}$, to minimize measurement condition effects and to represent the measurement accuracy, respectively. In what follows, we briefly review the basic definitions. The local misorientation, $M_{\mathrm{L}}$, can be defined as

$$
M_{\mathrm{L}}(i)=\frac{1}{6} \sum_{j=1}^{6} m(i, j),
$$

where $m(i, j)$ denotes the misorientation angle between two orientations $i$ and $j$. The sum runs over all first-neighbor sites (located at a distance $h=h_{0}, h_{0}$ being the measurement step size). The number of first neighbors depends on the grid

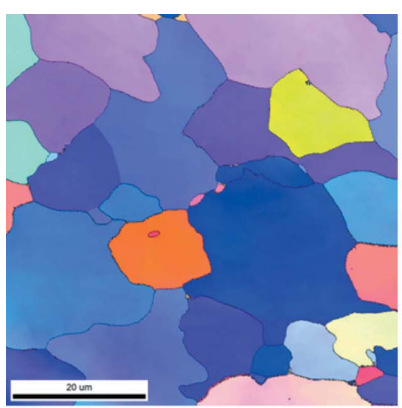

AR

Average $\mathrm{Cl}: 0.73$

Average IQ: 5732.20

Average KAM: 0.364

diamet $\left(d_{2} / d_{0}\right)$ define the major and minor strains, respectively, where $d_{0}$ is the initial strain ratio are defined as $\bar{\varepsilon}=\left(\varepsilon_{\mathrm{I}}^{2}+\varepsilon_{\mathrm{II}}^{2}\right)^{1 / 2}$ and $\rho=\varepsilon_{\mathrm{II}} / \varepsilon_{\mathrm{I}}$, respectively.

an EBSD detector. The EBSD detector is an EDAX (TSLOIM), with a phosphor screen and a DigiView CCD camera. A forward scatter detector was attached under the screen. The microscope was operated at $20 \mathrm{keV}$ and had a working distance of $15 \mathrm{~mm}$. Crystal orientation data were acquired over a hexagonal grid with a spacing $\left(h_{0}\right)$ of $0.1 \mu \mathrm{m}$. Table 2 summarizes the scan areas, deformation states and average grain-size distributions for each deformation condition.

Fig. 2 displays inverse pole figure maps corresponding to scans \#5, \#7, \#13 and \#18 as standard (ND) maps, where ND designates the normal direction of the sheet plane. For the EBSD analysis, a standard grain recognition procedure was used, with a misorientation angle threshold set to $5^{\circ}$ (Bachmann et al., 2011; Schwartz et al., 2000). Detailed information for each EBSD map including average confidence index (CI) and average image quality (IQ) is provided in the supplementary material (Table S1) and it renders any cleanup procedure unnecessary.

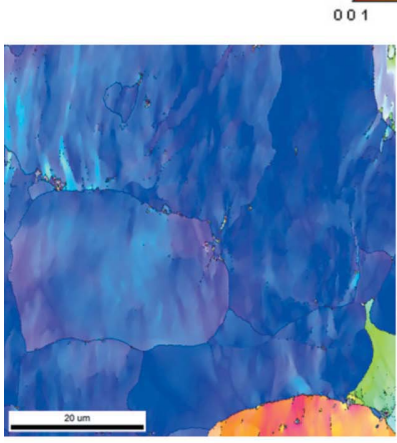

PS

Average $\mathrm{Cl}: 0.64$

Average IQ: 3205.62

Average KAM: 0.605

Figure 2

Inverse pole figure orientation maps for the AR material after different loading paths (UAT, PS and EBA stretching). ND represents the out-ofplane direction; the horizontal and vertical directions of the maps are the rolling and transverse directions of the original sheet, respectively. Step size $h_{0}=0.1 \mu \mathrm{m}$. Black lines correspond to boundaries with misorientations exceeding $15^{\circ}$. Corresponding IQ maps are plotted in the supplementary material (Fig. S1). 
pattern type used in the acquisition process [i.e. four points for a square grid and six points for a hexagonal grid, as applied in equation (1)]. Misorientation angles $m(i, j)$ are only taken into account if pixels $i$ and $j$ lie within the same grain. The local misorientation magnitude can be extended to the following nearest-neighbor kernels. In the case of the hexagonal grid, the five kernel sizes are $h_{0}, 3^{1 / 2} h_{0}, 2 h_{0}, 7^{1 / 2} h_{0}$ and $3 h_{0}$, with six neighbors at each distance, except for the fourth kernel which has 12 members (see Fig. S2 in the supplementary material). An extended expression of equation (1) is given by

$$
M_{\mathrm{L}}^{(k)}(i)=\frac{1}{\operatorname{pts}(k)} \sum_{j=1}^{\mathrm{pts}(k)} m\left(i, j^{(k)}\right),
$$

where $k$ equals 1 for the nearest-neighbor kernel, 2 for the second-neighbor kernel, and so on. The slope of the regression line for the first five values of $M_{\mathrm{L}}^{(k)}($ i.e. $k=1, \ldots, 5), \mathrm{d} M_{\mathrm{L}}^{(k)} / \mathrm{d} h$, is defined as the local gradient of the crystal orientation $G_{\mathrm{L}}$. This magnitude is a measure of the heterogeneity of the orientation. The higher the value, the more the orientation changes over a given distance, resulting from a higher local density of defects producing these misorientations. It should be noted that $k=5$ has been estimated as an adequate value in this study. A higher $k$ value leads to slightly higher values of $G_{\mathrm{L}}$ and an unnecessary increase in the computational cost;
Fig. S3 in the supplementary material shows this effect with respect to the local gradient misorientation in the case of $k=8$.

The intersection value at $h=0$ defines the local background noise $B_{\mathrm{L}}$. The background noise represents the accuracy of the EBSD technique, typically in the range of $0.5^{\circ}$ for modern systems, but the accuracy depends on many parameters, including the crystal orientation itself and the presence of defects. Fig. 3 shows the local gradient maps, for $60 \times 60 \mu \mathrm{m}$ areas taken from scans \#5, \#7, \#13 and \#18 (see Table 2). The maps were calculated using in-house software written in Fortran. The grain boundaries are superimposed (white lines correspond to boundaries with misorientations exceeding $15^{\circ}$ ) to facilitate the visualization of the microstructure. A single value averaged over all areas measured within a given sample, $G_{\mathrm{L} \text { ave }}$, represents the magnitude of the orientation gradient in a given state. In sample $\mathrm{AR}, G_{\mathrm{L} \text { ave }}$ is approximately $0.3^{\circ} \mu \mathrm{m}^{-1}$.

Owing to the deformation process and the heterogeneity of plastic deformation, scans \#7 and \#13 show an increase in the number of zones where misorientations localize appreciably. High values of $G_{\mathrm{L}}$ are observed mainly near the grain boundaries, but are also seen inside and across the grains. Moreover, there are some regions, even including multiple grains, where misorientations are localized. As will be discussed later, and as Vachhani et al. (2016) recently observed
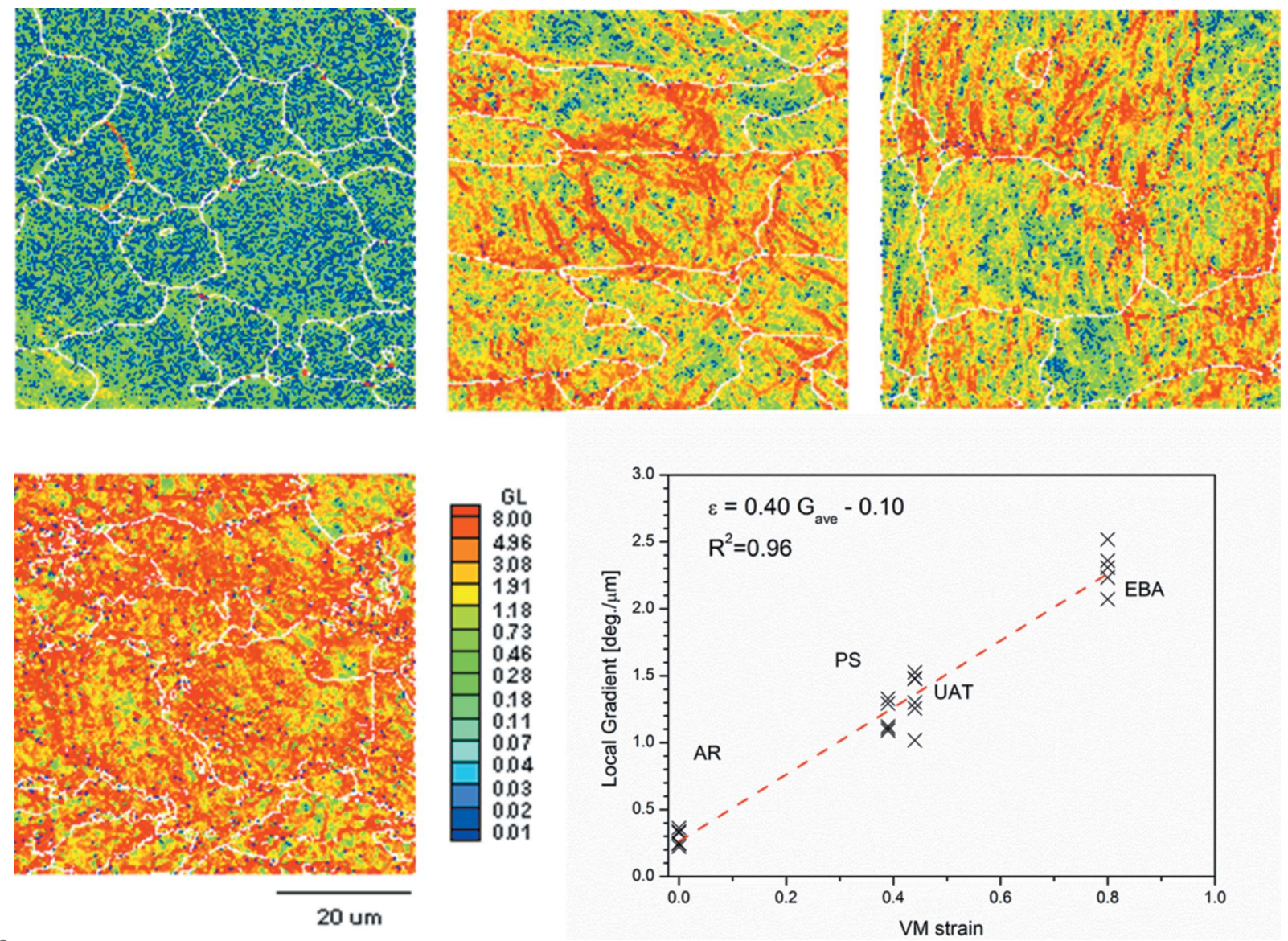

Figure 3

Maps of local gradients of crystal orientations for the AR, UAT, PS and EBA samples and average values for the measured EBSD maps as a function of the equivalent plastic strain. White lines correspond to boundaries with misorientations exceeding $15^{\circ}$. 
in high-purity aluminium, the local misorientation near the grain boundary does not show a simple correlation with the misorientation between crystals on either side of the GB zone. Also, Thorning et al. (2005) claim that differences in geometrically necessary boundary structures among the subgrain domains are not a key indicator of grain-interaction effects. In that study, the authors investigated $\mathrm{Cu}$ samples, with grain sizes of approximately $100 \mu \mathrm{m}$, deformed in tension. As might be expected, a similar analysis that accounts for a higher concentration of local gradients can be done using scan \#18, which was taken after a von Mises strain of $\sim 0.80$. Note that the majority of GBs accumulate local misorientations.

It is interesting that there are no qualitative differences if the local gradient of crystal orientation is replaced by the KAM values calculated in the present analysis. This is illustrated by comparing the maps in Figs. 3 and 4.

Furthermore, the extent of plastic deformation is an important indicator of the material's susceptibility to crack initiation, crack growth or grain fragmentation. Since direct mesoscopic scale strain measurement is difficult, investigators have considered methods to correlate the accumulated strain with the polycrystalline aggregate orientation field (Kamaya $e t$ al., 2005; Schayes et al., 2016; Yoda et al., 2010). Kamaya (2011) estimated the plastic deformation based on the average of the local orientation gradient, $G_{\mathrm{L}}$, because this parameter is less sensitive to measurement conditions. It is possible that this relationship remains valid even when considering different loading paths. The plots in Figs. 3 and 4 show the averages of $G_{\mathrm{L}}$ and KAM for all measured EBSD maps as a function of the effective strain determined from the grid pattern. A spread in values is observed, which is associated with the sampling. A linear-regression analysis for parameters $G_{\mathrm{L} \text { ave }}$ and $\mathrm{KAM}_{\mathrm{ave}}$ confirms that a linear fit is a reasonable first approximation for the entire data range. Correlation coefficients $\left(R^{2}\right)$ of 0.96 and 0.92 were calculated for $G_{\mathrm{L} \text { ave }}$ and $\mathrm{KAM}_{\text {ave }}$, respectively, and the following relations were obtained:

$$
\begin{gathered}
\bar{\varepsilon}^{G}=0.40 G_{\mathrm{Lave}}-0.10, \\
\bar{\varepsilon}^{K}=1.81 \mathrm{KAM}_{\mathrm{ave}}-0.62 .
\end{gathered}
$$

It would be desirable to extend equations ( $3 a)$ and ( $3 b)$ by replacing average magnitudes with local ones, in order to estimate the grain's average plastic strain. However, as we shall see below, this is not possible in a quantitative form and the relations can only be used to give a rough estimate of the average strain in the grain. Fig. 5 shows the estimated grainaverage plastic strains calculated using either the local gradient or the KAM as the reference [i.e. in equation (3) the values $G_{\text {ave }}$ and $\mathrm{KAM}_{\text {ave }}$ are replaced by $G_{\mathrm{L}}$ and KAM averaged over the grain domains]. When these cases are

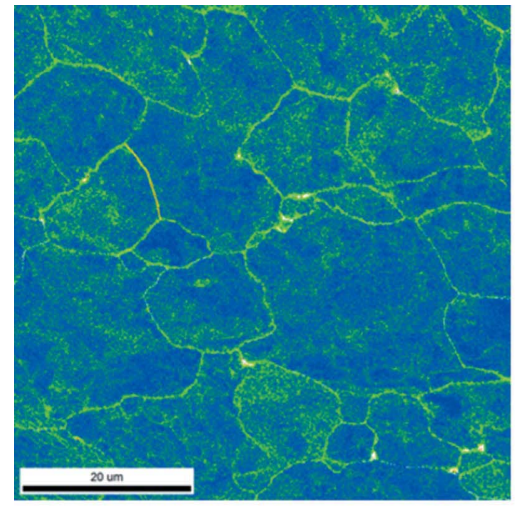

AR

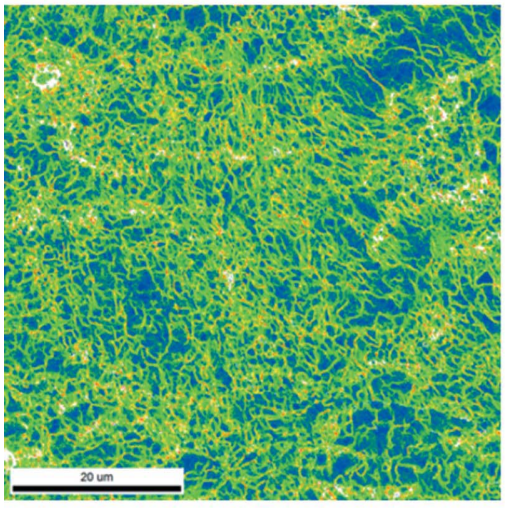

EBA

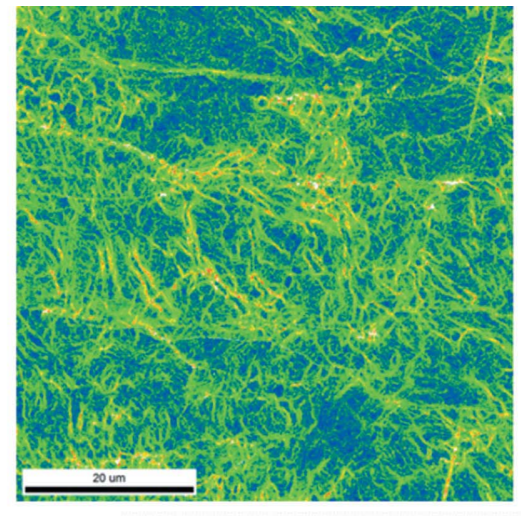

UAT

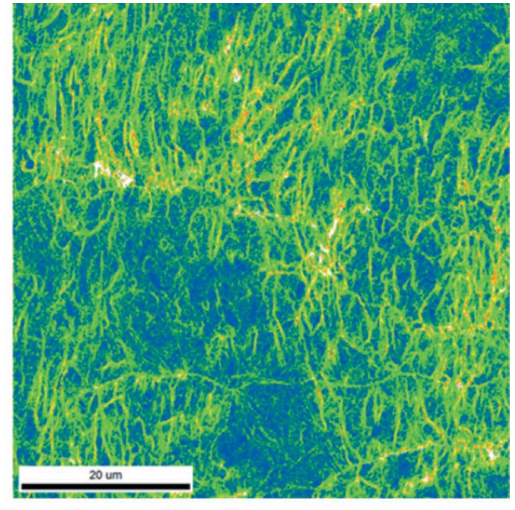

PS
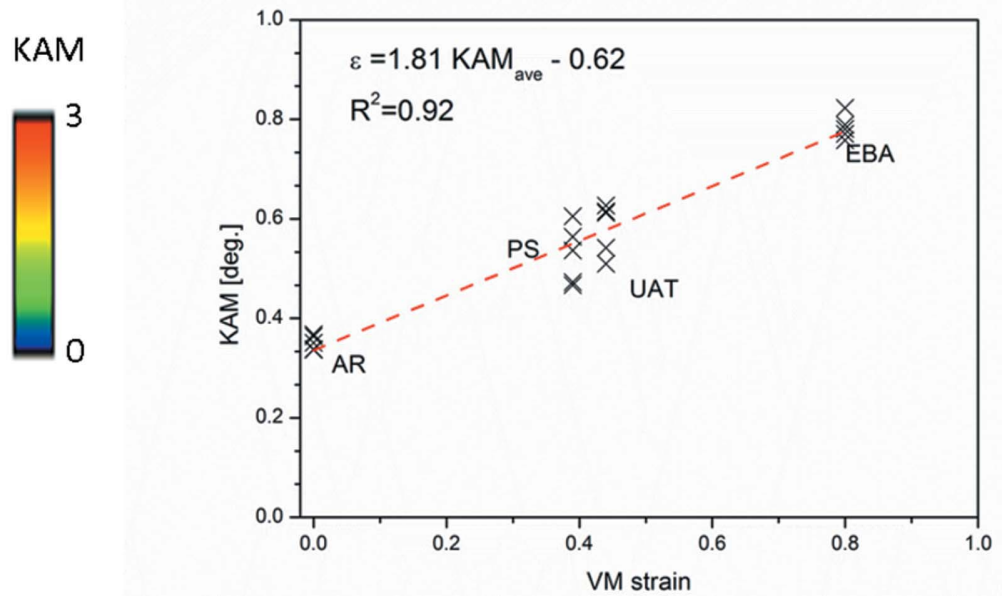

Figure 4

Maps of kernel average misorientation of crystal orientation for the AR, UAT, PS and EBA samples and average values for the measured EBSD maps as a function of the equivalent plastic strain. 
compared, the dispersion in the estimated grain-average plastic strain increases as the grain size decreases. This behavior is illustrated for one of the UAT scans (\#7), but it was systematically verified on all measured EBSD maps. The poor correlation confirms that equations $(3 a)$ and $(3 b)$ only make sense from a qualitative point of view at the grain level. However, if only the solid black circles in Fig. 5 are considered (i.e. grain sizes $>10^{4}$ pixels), the Pearson correlation factor reaches 0.78 with a slope of 0.95 , which indicates that grain strains based on grain-average values of $G_{\mathrm{L}}$ and KAM correlate well, and it could make sense to use them to roughly estimate the strain at the grain level. It follows that the calibrated values of $G_{\mathrm{L} \text { ave }}$ or $\mathrm{KAM}_{\mathrm{ave}}$ can be used in equations (3) to assess the equivalent plastic strain in an average sense only.

\subsection{Grain boundary zone - a model for the linear} misorientation profile and the introduction of new EBSD parameters: $\lambda, \beta$, BET, GAS

In a recrystallized IF steel sheet with a mean grain size of $16 \mu \mathrm{m}$, Allain-Bonasso et al. (2012) showed different misorientation profiles, after $17 \%$ uniaxial deformation. They also observed that misorientation profiles taken from the grain interior show an approximately linear orientation gradient when approaching the grain boundary (see Fig. 3 of AllainBonasso et al., 2012). Kashihara \& Wert (2006) reported the presence of orientation gradients near the GB in an aluminium bicrystal under tension, as well.

In this section, we seek to determine the fraction of the grain boundary zones that can, on average, be described with a linear orientation-gradient profile and to obtain EBSDderived parameters describing this behavior. Results obtained for different deformation conditions will be compared.

In order to verify the existence of local orientation gradients close to the GB, a GB zone must first be defined. In the following, this area includes all pixels belonging to the grain

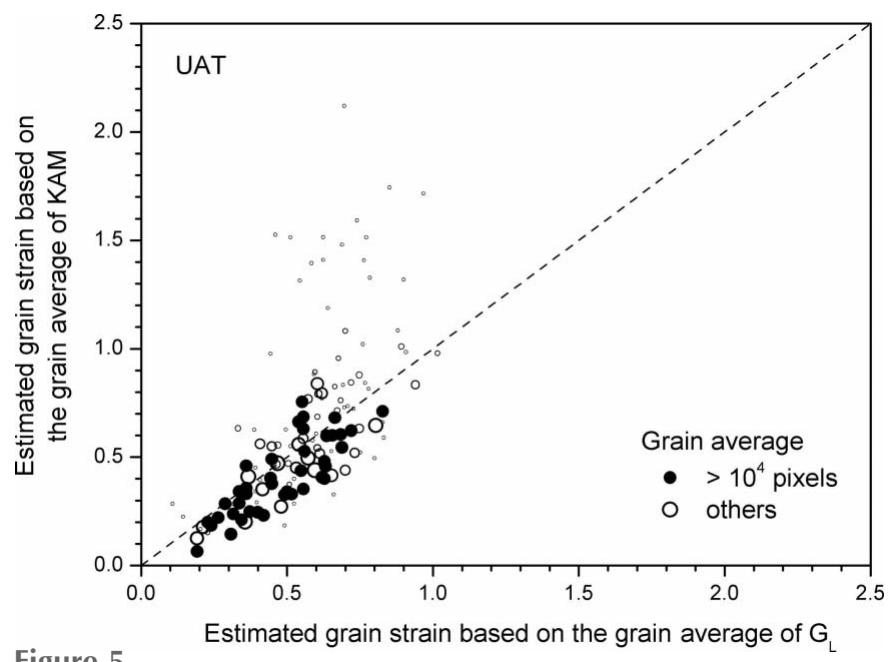

Figure 5

Estimation of the grain-average plastic strain using both the grain average of the local gradient of crystal orientation and the KAM parameter. The symbol size for grains smaller than $10^{4}$ pixels is proportional to the apparent grain diameter. that are within $5 \mu \mathrm{m}$ of a given GB and no closer to any other GB. The orientation profiles were obtained by taking the average local misorientation over $0.2 \mu \mathrm{m}$ thick bins within this GB zone and at increasing distances from the GB (i.e. all pixels belonging to the analyzed GBZ and their distances to the grain border are between $d_{i}$ and $\left.d_{i}+0.2 \mu \mathrm{m}\right)$. The generic procedure used in order to determine the GBZ is as follows.

Given all the pixels belonging to a given grain $k$ :

Step 1. Determine the set of pixels that are located at the GB (i.e. pixels with first neighbors that do not belong to the same grain).

Step 2. Separate the set of GB pixels into subsets $\mathrm{GB}_{i}$, one subset for each border with a neighboring grain.

Step 3. Determine the GBZs. The pixel ( $r$ ) belongs to the $\mathrm{GBZ}_{i}$ of subset $\mathrm{GB}_{i}$ if the pixel is closest to a pixel in the $\mathrm{GB}_{i}$ subset and it lies within a fixed threshold distance. This test is repeated for all pixels in the grain.

The application of this simple model is shown in Fig. 6, where the analysis is applied to the highlighted GB zones in scan \#13. Orientation gradients associated with GB zones I, II and IV exhibit approximately linear behavior over a traverse from the GB to the grain interior, while in case III, no misorientation gradient is observed near the GB.

Fig. 6(c) shows several examples of misorientation line profiles at two locations along the analyzed GBs. In the case of GB I, the two point-to-point misorientation lines show more pronounced variations at distances close to the GB while the variations are less pronounced at distances greater than $2 \mu \mathrm{m}$, which is consistent with the average profile shown in Fig. 6(b). For GB II, the misorientation profile presents even more variations depending on the place from which it was extracted. This is consistent with the high concentration of low-angle boundaries on the side of the GB where the traverses were made, as seen in Fig. 6(a). The point-to-GB misorientation reaches its maximum at a distance of $2 \mu \mathrm{m}$ from this GB. A similar situation arises in several other profiles checked along this GB. Clearly, the misorientation profile associated with GB III has a different behavior, where point-to-point misorientations vary little from an average value of $0.4^{\circ}$. Misorientations along traverses originating at GB IV were similar to cases I and II but with smoother variations.

Linear misorientation profiles close to boundaries can be characterized by two parameters, $\lambda$ and $\beta$, as shown in Fig. $6(b)$. The value of $\lambda$ should be considered as an effective thickness over which a constant orientation gradient occurs, and $\beta$ is taken as a measure of the severity of that gradient. The parameter tangent $(\beta)$, in units of ${ }^{\circ} \mu \mathrm{m}^{-1}$, is the slope of the observed misorientation profile, i.e. the magnitude of the gradient. These parameters were determined from a leastsquares fitting of the experimental data from two-dimensional sections. A more extensive analysis would require the measurement of three-dimensional microstructures, which is beyond the scope of the present work. Additionally, because local misorientations may generate important uncertainties in close proximity to the grain boundaries due to the overlapping of Kikuchi patterns, for example, a gap of $0.5 \mu \mathrm{m}$ was imposed. This avoided high local variations in the misorientation profile. 
As already mentioned, the variations in local crystal orientation were analyzed over a distance of up to $5 \mu \mathrm{m}$ from the GB. Experimental profiles, even in this range of $5 \mu \mathrm{m}$, can show strong variations of local misorientations, and as a consequence, there are important variations in the $\lambda$ and $\beta$ parameters depending on which GB is selected. Therefore, in order to validate this analysis, it was necessary to evaluate how many of the GB zones fit the proposed model. The model profile is considered acceptable when the square of the correlation coefficient of the least-squares fitting procedure, $R^{2}$, is greater than 0.75 , as shown in Fig. $6(b)$, GB I. It should be noted that the threshold value of 0.75 was selected arbitrarily. Supplementary Fig. S4 shows the effects of decreasing this threshold to $R^{2}>0.60$ or increasing it to $R^{2}>0.90$. At distances greater than $\lambda$, the misorientation profile will be characterized by its deviation from the average value using the $L_{\infty} \operatorname{norm}\left[\right.$ i.e. $\left.\left\|\mathbf{x}_{\infty}\right\|=\max \left(\left|x_{1}\right|, \ldots,\left|x_{n}\right|\right)\right]$.

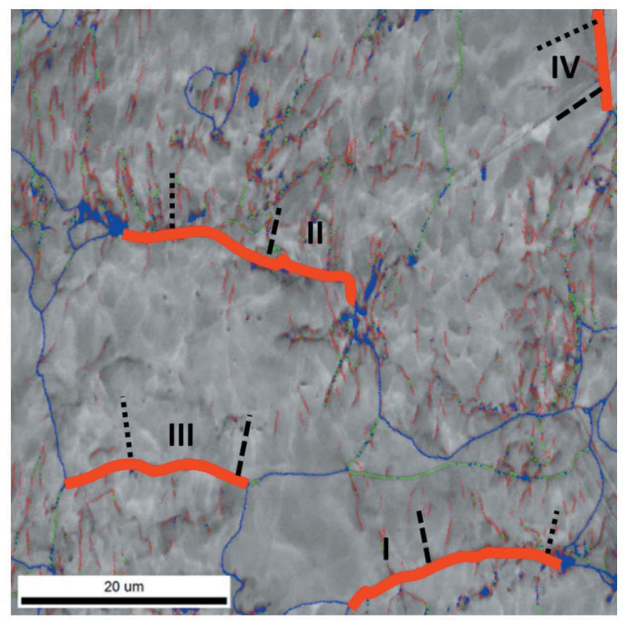

(a)
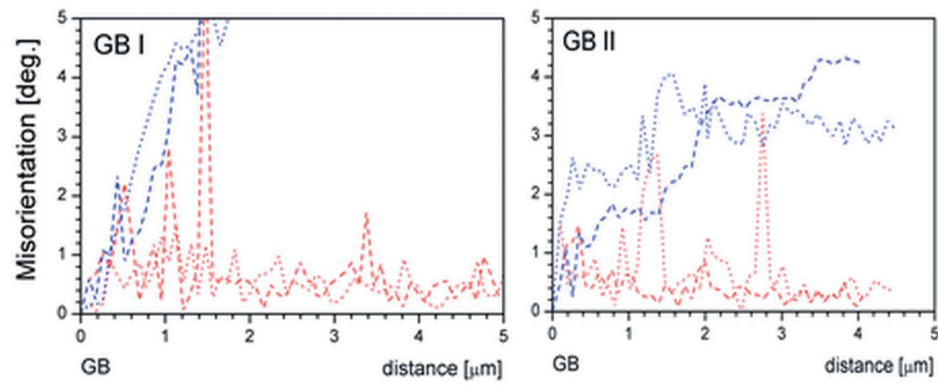
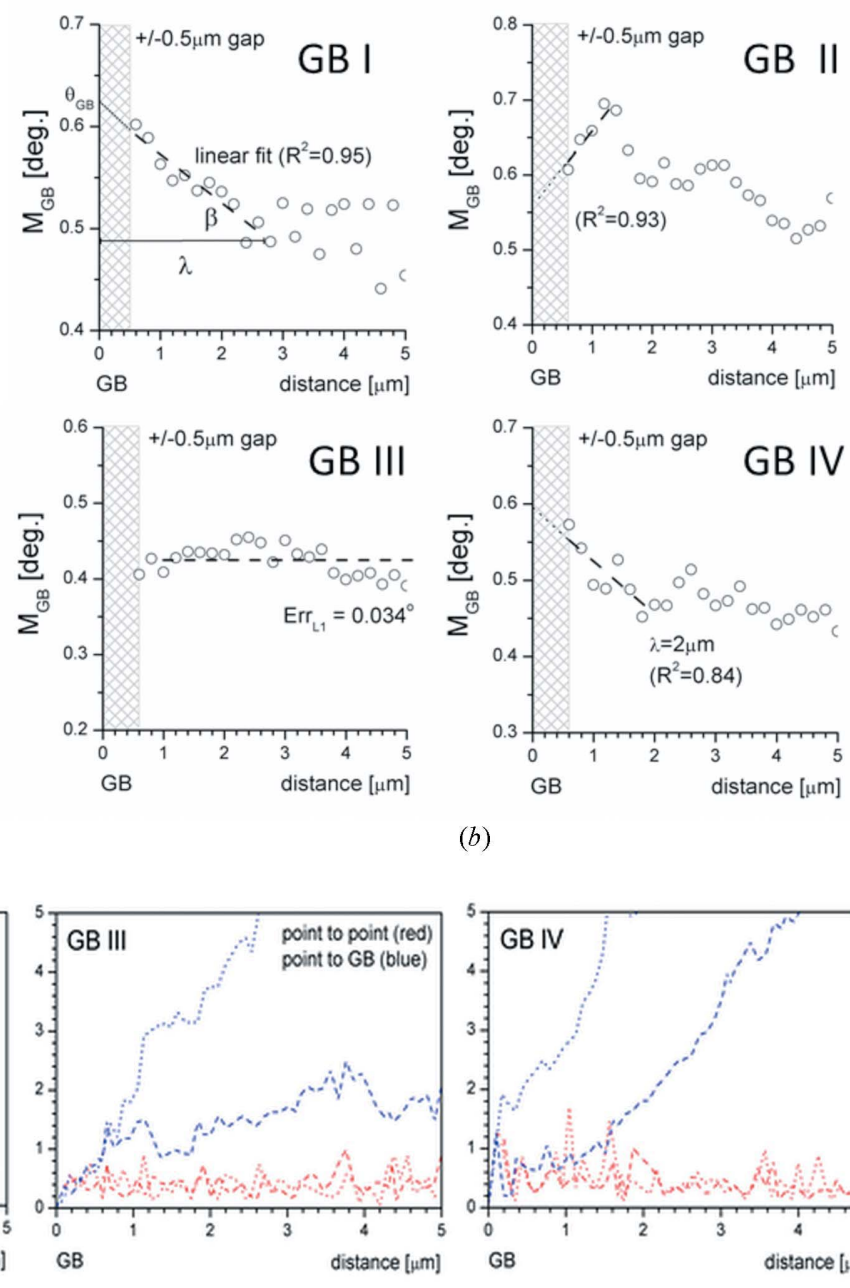

(b)

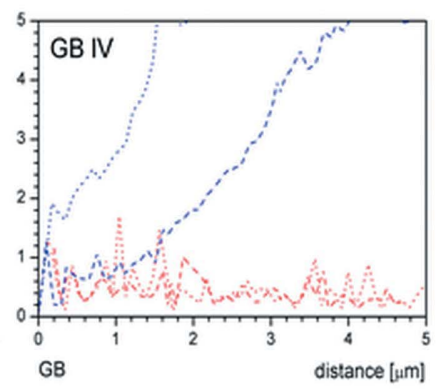

(c)

The following results are based on the GB zones in which the model profile was verified $\left(R^{2}>0.75\right)$. A total of $6436 \mathrm{~GB}$ profiles were evaluated. In a zone of width $\lambda$ from the GB, statistical analysis showed that about $50 \%$ of the analyzed near-GB misorientation profiles could be described as lines with a negative slope. That proportion decreases with increasing deformation: AR (69\%), UAT (57\%), PS (58\%) and EBA $(47 \%)$. It is interesting that uniaxial tension and plane strain samples deformed to similar equivalent strains contained a similar fraction of GBs with a linear profile (i.e. constant gradient). This does not mean that for all deformation conditions the observed gradients are the same; in fact, as the deformation increased, the misorientation in the GB zones and thus the severity of the gradient (i.e. $\beta$ value) increased acordingly. There is a trend for the misorientations to be profile, although the model is far from describing entirely the

Figure 6

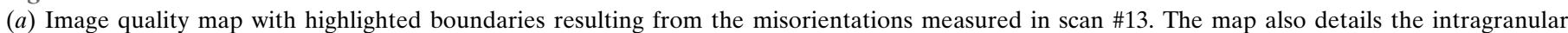

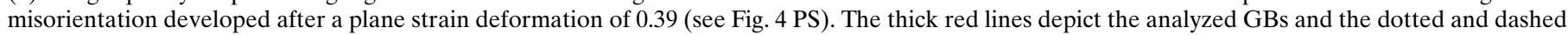

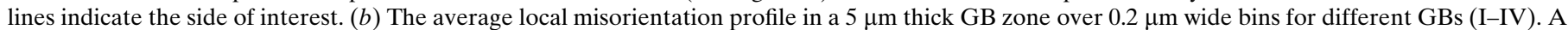

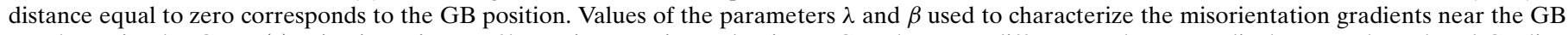

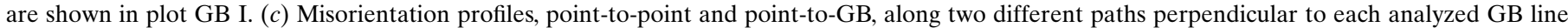
[black dotted and dashed lines in $(a)$ ]. 

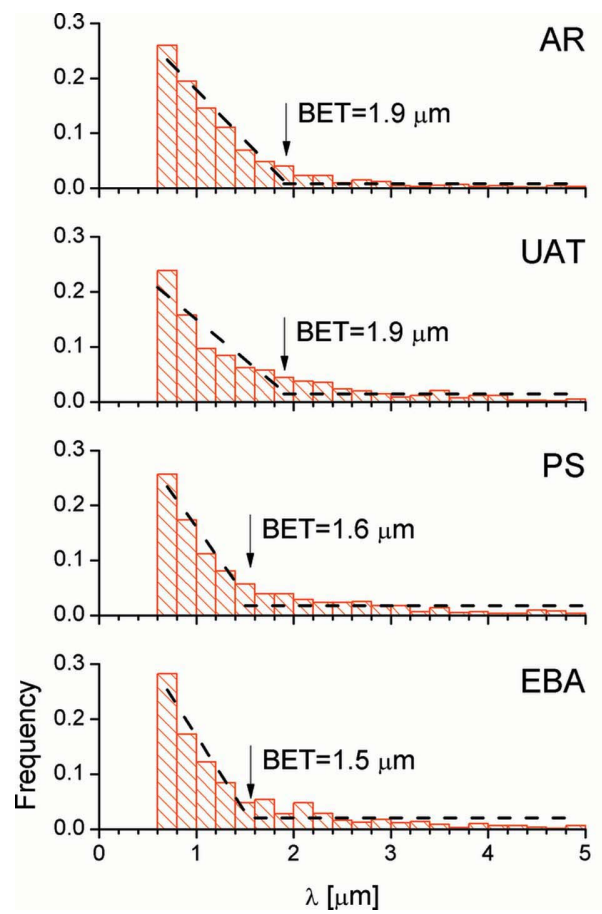

EBA

Figure 7

Distribution of effective thickness $\lambda$ (left) and $\lambda / D$ (right) for all admissible GB profiles based on the average of local misorientations.

developed microstructure's complexity. It is clear that the results of this analysis must be interpreted only as a first approximation of a complex phenomenon. Two other issues arise from this analysis: firstly, GB profiles that do not fulfill the $R^{2}>0.75$ condition (Fig. $6 b$, case of GB II); and secondly, the behavior of the misorientation profile at distances greater than $\lambda$ in those GB zones where a linear gradient is observed (Fig. 6b, case of GB I). A segment of the misorientation profile will be assumed flat if the maximum deviation from the average value does not exceed a threshold of $0.05^{\circ}$. The segment has a length $[\lambda, 5 \mu \mathrm{m}]$, where $\lambda$ can be as small as the $0.5 \mu \mathrm{m}$ bin width, and thus these segments exhibit an almost constant crystal orientation over a distance $\lambda$ from the grain boundary (Fig. $6 b$, case of GB III). The fraction of orientation profiles that fulfilled this flatness condition varied with deformation state: for the as-received, uniaxial, plane strain and biaxial specimens, 32, 11, 15 and 3\%, respectively. As deformation increases, flat misorientation profiles at the grain interiors are less frequent, which is consistent with the qualitative information provided by the inverse pole figures shown in Fig. 2 and the image quality maps included in the supplementary material (Fig. S1).

The histograms in Fig. 7 summarize the $\lambda$ parameter distributions obtained from all admissible $\left(R^{2}>0.75\right) \mathrm{GB}$ zones. Allain-Bonasso et al. (2012) demonstrated that $\lambda / D$ rather than $\lambda$ is approximately constant. Examining the distributions of the $\lambda$ parameter and the magnitudes of the scaled value $\lambda / D$ ( $D$ is the grain diameter) in our measurements, we found that the assumptions of constant $\lambda$ or $\lambda / D$ are not valid for describing the local gradients developed near
(2013). In order to quantify the BET value, a bi-linear procedure was used (dashed lines in Fig. 7, on the left-hand side). The resulting BET values are 1.9, 1.9, 1.6 and $1.5 \mu \mathrm{m}$ for the AR, UAT, PS and EBA conditions, respectively. These BET values indicate the existence of an effective GB thickness.

In order to assess a possible influence of grain size on the development of the near-GB orientation gradients, the data were split into two categories, based on a grain diameter

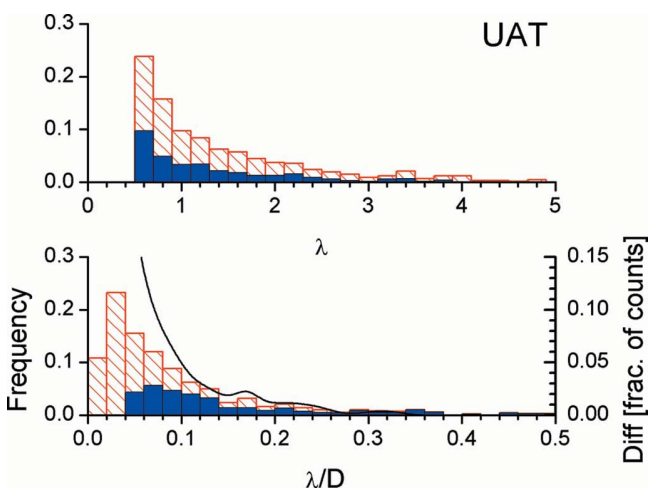

Figure 8

Distribution of effective thickness $\lambda$ (top) and $\lambda / D$ (bottom) for all admissible GB profiles based on the average of local misorientations after UAT. Striped and solid bars representing grains with an apparent diameter greater than and less than $15 \mu \mathrm{m}$, respectively, identify the fraction of GB profiles with a given value of $\lambda$ or $\lambda / D$. The lack of cases for values of $\lambda / D$ less than 0.04 is due to the presence of a gap of $0.5 \mu \mathrm{m}$ in the definition of $\lambda$. The solid line in the bottom graph indicates the difference between the two populations in terms of fraction of counts per bin. 
threshold $D^{*}$ of $15 \mu \mathrm{m}$ (see the grain-size distribution histograms on the right-hand side of Table 2). In other words, the counts that contribute to each bin of $\lambda$ and $\lambda / D$ histograms have been split into two populations corresponding to grains with $D<D^{*}$ and $D>D^{*}$. Both populations showed a similar distribution of counts in terms of $\lambda$, but not with respect to $\lambda /$ $D$. Fig. 8 shows these two populations for the case of UAT. The difference between the bin frequencies of the two $\lambda / D$ populations is clearly seen in the bottom graph of Fig. 8. All deformation states, including AR, exhibited similar trends and these results are plotted in the supplementary material (Fig. S5). These histograms show that there are no significant differences that result from variations in grain size.

Fig. $9(a)$ complements these results. It shows the histograms corresponding to the values of misorientation $\theta_{\mathrm{GB}}$ extrapolated from parameters $\lambda$ and $\beta$, obtained from all admissible GBZ profiles, at the GB (i.e. the intercept of the line, which characterizes the misorientation gradient, at the GB; see Fig. $6 b$ case I). As deformation increases, the orientation
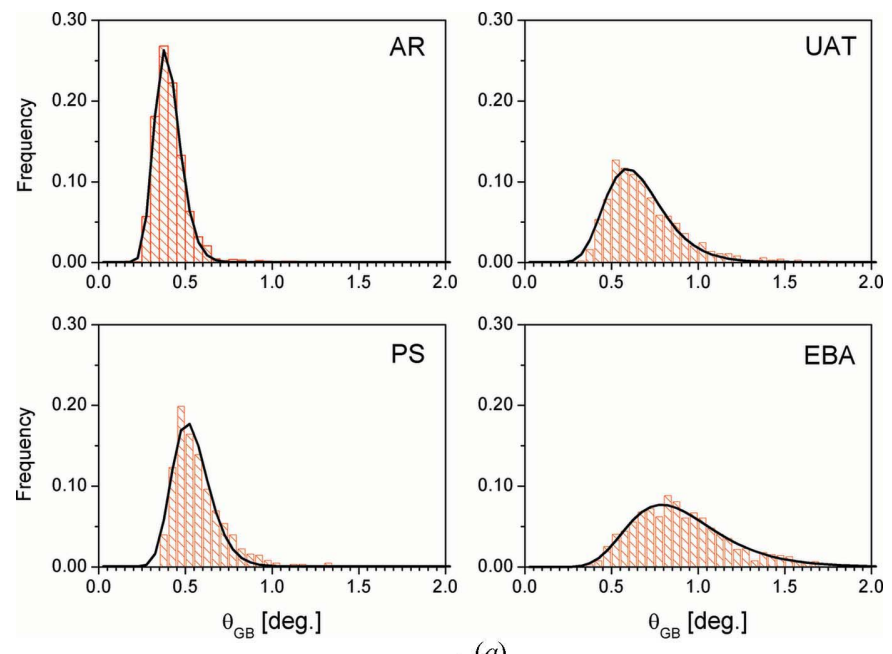

(a)

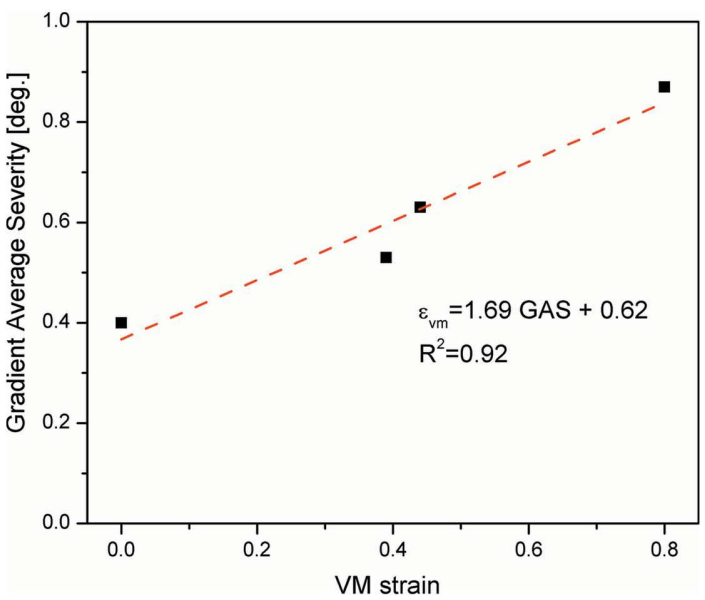

(b)

Figure 9

(a) Distribution of the extrapolated misorientation at GBs for the set of GBZs that show a linear near-GB misorientation profile. Histograms take into account all measured EBSD maps for each loading condition. (b) Correlation between the peak of the distribution, GAS, and the measured equivalent plastic strain. gradients (and thus $\theta_{\mathrm{GB}}$ values) rise as well. Fig. $9(b)$ shows that the $\theta_{\mathrm{GB}}$ value at which the distribution peak occurs could also be used as a gauge of the strain. We will use this value to characterize the intensity or severity of the gradient in the GBZ, calling it gradient average severity (GAS). These two new EBSD-based parameters, BET and GAS, should be considered together and seen as a first statistical approximation describing the orientation gradients in the GBZ.

\subsection{Comparison of orientation gradients between GBZ and grain-core zone}

In order to quantify the development of local crystal orientation gradients, all pixel orientations associated with a given deformation state (i.e. AR, UAT, PS or EBA) were classified as belonging to a grain boundary zone or belonging to a core zone. Two alternatives can then be used, as illustrated in Fig. 10(a): either the $\lambda_{i}$ value is calculated for each GBZ or an effective thickness is defined for all GBs. Each reliable pixel orientation determined by EBSD is assigned to the nearest grain boundary. If the distance from the pixel to the nearest boundary is shorter than a specified thickness, the pixel is considered to belong to the GBZ. If not, it is
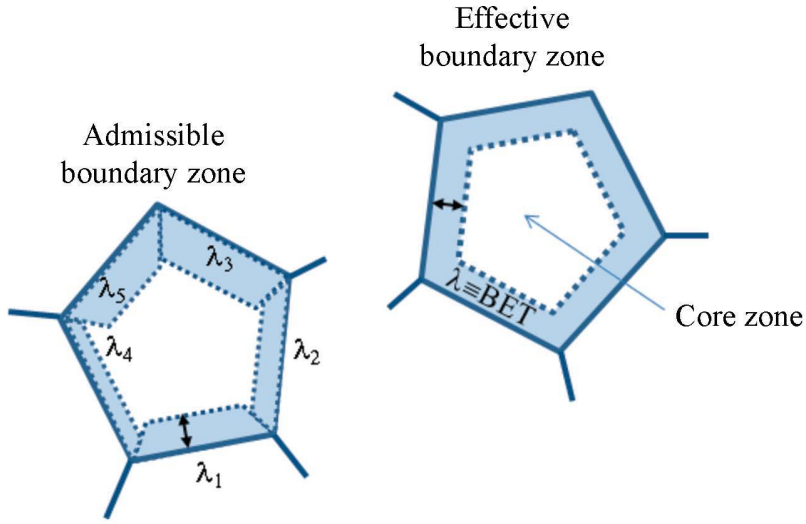

(a)

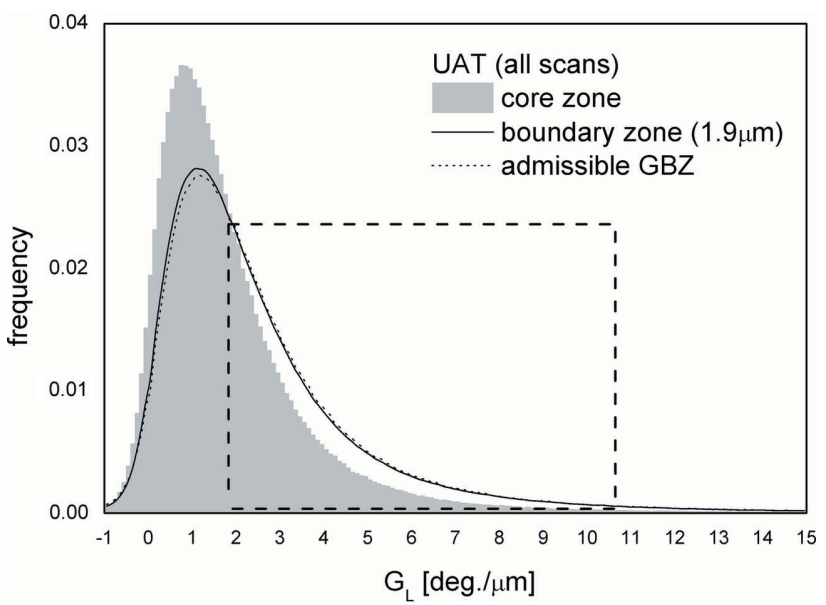

(b)

Figure 10

(a) Definition of GBZs and core zones. (b) Example of the distribution of the local gradient of crystal orientations in core and grain boundary zones of all of a sample's grains. 
Table 3

Most frequent values of the distribution of the local gradient of crystal orientation $\left({ }^{\circ} \mu \mathrm{m}^{-1}\right)$, associated with the core and grain boundary zones.

\begin{tabular}{lllll}
\hline & AR & UAT & PS & EBA \\
\hline GB zone & 0.20 & 1.15 & 0.95 & 2.35 \\
Core zone & 0.20 & 0.80 & 0.75 & 2.35 \\
\hline
\end{tabular}

considered to be a core-zone point. The analysis is made for each grain. If a grain does not have an admissible GBZ, for the purpose of defining the distribution of local gradients of crystal orientations, all pixels are considered to belong to the core.

Fig. 10(b) illustrates the distributions of the local gradient of crystal orientation in the grains for both the admissible or the effective grain boundary zone and grain-core zones obtained from all uniaxial-tension EBSD scans. Negligible differences were found between the two definitions of a GBZ (Fig. 10a), and only a few grains did not have an admissible GBZ. This result makes clear the acceptability of using the effective boundary zone approximation in order to account for the differences between the GB and core zones, at least from a statistical view point.

For the case of an effective boundary zone, a BET value of $1.9 \mu \mathrm{m}$ was assumed (this is consistent with the results plotted in Fig. 7). All $G_{\mathrm{L}}$ distributions show a single peak with a maximum value that depends on the material state and on the type of zone considered (values of $G_{\mathrm{L}}$ are given in Table 3 for the AR state and for all three loading conditions). For the UAT case, the most frequent of the $G_{\mathrm{L}}$ values in the GB zones $\left(1.15^{\circ} \mu \mathrm{m}^{-1}\right)$ exceeds the value obtained from the core zones $\left(0.80^{\circ} \mu \mathrm{m}^{-1}\right)$. Moreover, the GB zones yield a greater proportion of high $G_{\mathrm{L}}$ values, when compared to the corresponding core zones [see the dashed box in Fig. 10(b)]. The positive difference between these values quantifies the preferential development of the local crystal orientation gradient near the grain boundaries. After taking a linear fit of these values (Table 3 ), the results can be plotted versus the equivalent deformation developed by each experiment in the grain boundary and core zones. A fit of this representation by linear regression gives $\bar{\varepsilon}^{\mathrm{G}}=0.37 f^{\mathrm{GB} \text { zone }}-0.026$ and $\bar{\varepsilon}^{\mathrm{G}}=0.38 f^{\text {core zone }}-0.022$. These slopes are nearly the same as that obtained previously from the average deformations [equation (3a)]. In the AR case both distributions are similar; that is compatible with the homogeneity observed in the different EBSD maps (scans \#1-6). On the other hand, as mentioned previously, admissible GBZs are also present in the AR condition, but with a low GAS of $0.4^{\circ}$. The fact that the distributions are similar means that localization of the local gradient of crystal orientations has not developed sufficiently to make a notable difference with the local gradient obtained in the core zone. The plane strain results are very similar to uniaxial tension, while after equal-biaxial deformation the most frequent values of $G_{\mathrm{L}}$ are the same in both types of zones. This lack of difference is consistent with a more homogenous development of the microstructure at an accumulated strain of $\sim 0.8$, where the internal microstructure can be thought complex enough to make the distinction between GB and core zones unnecessary in terms of distribution features (see Fig. 3 and supplementary Fig. S1).

In addition, it was verified that, by restricting the calculation of the average value of $G_{\mathrm{L}}$ over the admissible GBZs (not shown in the paper), these values are in complete agreement with the slope and correlation coefficients obtained from data averaged over all pixel orientations in Fig. 3.

The influence of slip transfer on the development of crystal orientation gradients between contiguous GB zones was also analyzed (Fig. 11). The geometry of the slip transfer across a GB is usually characterized by two angles, $\varphi$ and $\xi$, the angles between slip-plane normals or slip directions, respectively, of two slip systems on either side of a boundary. The symbols in Fig. 11 are related to the value of the factor $m=\cos (\varphi) \cos (\xi)$, evaluated using the mean orientation over each GB zone and assuming that plastic deformation occurs by 24 slip systems of the types $\{110\}\langle 111\rangle$ and $\{112\}\langle 111\rangle$. The values of $m$ are between $\cos ^{2}\left(10^{\circ}\right)$ and 1 , between $\cos ^{2}\left(15^{\circ}\right)$ and $\cos ^{2}\left(10^{\circ}\right)$, and less than $\cos ^{2}\left(15^{\circ}\right)$. The star symbol in each plot corresponds to the GB with the highest degree of coplanarity of slip systems (i.e. $m$ close to 1 ). In the deformed samples, these particular GBs developed similar orientation gradients on both sides. It is clear from Fig. 11 that the local gradients averaged over contiguous GB zones $\left(G_{\mathrm{GB}}\right)$ do not correlate in a meaningful way with the $m$ factor. However, with respect to the most favorable case measured for each loading condition, both contiguous GB zones gave a magnitude roughly equal to
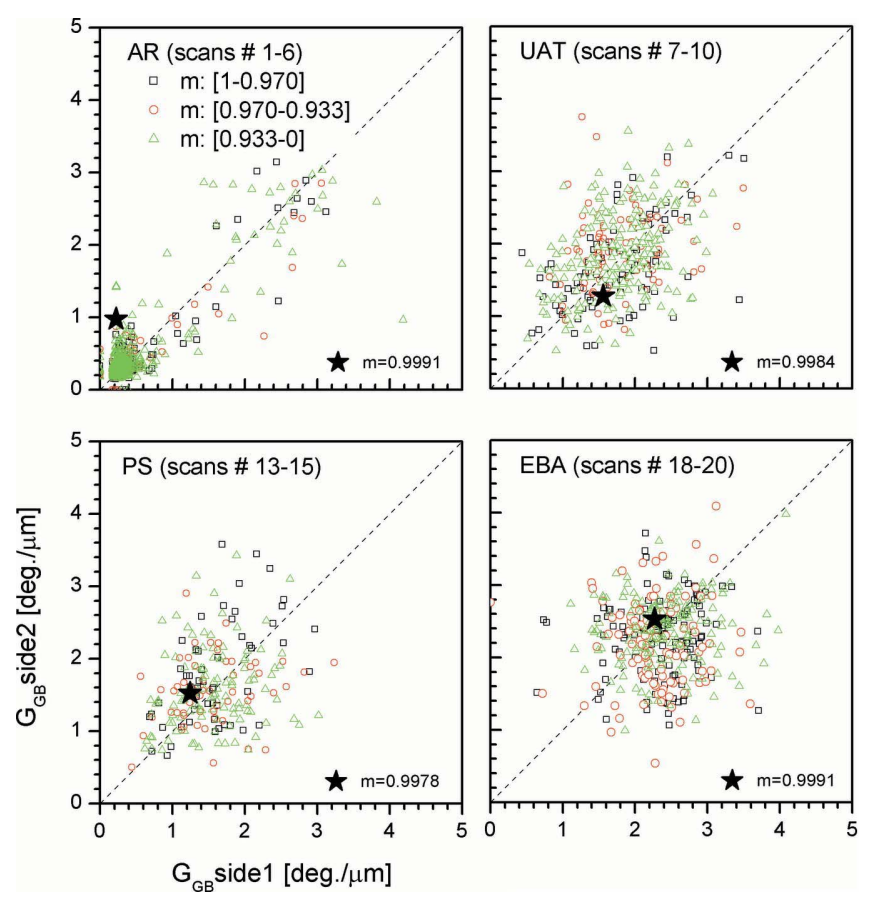

Figure 11

Correlation of the average local gradient of crystal orientations on both sides of the GB with the geometry of the slip transfer for conditions described by the $m$ factor: as received, uniaxial tension $(\bar{\varepsilon} \simeq 0.44)$, plane strain $(\bar{\varepsilon} \simeq 0.39)$ and equibiaxial stretching $(\bar{\varepsilon} \simeq 0.80)$. Assumed BET values are 1.9, 1.9, 1.6 and $1.5 \mu \mathrm{m}$ for AR, UAT, PS and EBA conditions, respectively. 
$G_{\mathrm{GB}}$. Consequently, in our application, which involves large strain, the $m$ value cannot be used as a key factor to characterize the localization of misorientations near the GB. Nevertheless, it is interesting to note that the distribution cloud showed sensitivity to the applied deformation and loading condition. If the analysis is restricted to an admissible GBZ (i.e. the GBZs on both sides of the GB are admissible), no qualitative difference is observed from that shown in Fig. 11. The number of contiguous GBZs that did not fulfill this more restrictive condition was between 30 and $40 \%$ of the analyzed GBs.

Finally, numerical results based on grain-scale crystal-plasticity models account for the presence of sharp strain gradients close to the GB (Bieler et al., 2009; Pouillier et al., 2012). In this sense, the incorporation of this type of analysis within the framework of a crystal-plasticity model might facilitate the understanding of the influence of grain boundaries in the formation, extension and strength of the observed intragranular and near-GB orientation gradients.

\section{Conclusions}

In the present paper, the plastic strain heterogeneity inside highly deformed grains and orientation gradients near grain boundaries have been analyzed and quantified through parameters derived from EBSD data. The analysis was performed on data from an AKDQ steel sheet deformed in uniaxial tension, plane strain and balanced-biaxial stretching. In all cases, EBSD mapping was performed in areas close to the necking zone.

The following conclusions can be drawn from these experiments and the EBSD measurements.

(1) The average of the local gradient of crystal orientations $G_{\mathrm{L} \text { ave }}$ and the KAM average can be used as macroscopic strain gauges, even when considering different loading paths. The calculated $G_{\mathrm{L} \text { ave }}$ and KAM average, in the AKDQ samples, show a linear correlation with the measured accumulated macroscopic strain, over the analyzed deformation range. However, neither of the linear fit equations are suitable for measuring the grain-average strain.

(2) It was found that about half of the evaluated GB zones, in all tested samples, have a local misorientation profile that can be considered linear. The analysis of misorientation profiles in the GBZs allowed the introduction of two new EBSD-based parameters, the BET (boundary effective thickness) and the GAS (gradient average severity), which are suitable for quantifying orientation gradients in the grain boundary zone. The GAS also correlates well with the macroscopic strain. The BET parameter has a value in the range of $1.5-2 \mu \mathrm{m}$, and it can be considered as a first approximation for quantifying the distance over which the grain boundary exerts influence for the AKDQ steel sheet.

(3) No simple correlation can be established between the averages of local gradients of crystal orientations over GB zones and the efficiency of the slip transfer between contiguous zones. Moreover, the observed dispersions of the average local misorientation in the GB zones are different for each loading condition. However, a high value of the factor $m$, close to 1 , seems sufficient to have similar values of $G_{\mathrm{GB}}$ on both sides of the GB.

\section{Acknowledgements}

The authors wish to thank the referees for their helpful comments and careful reading of this paper.

\section{Funding information}

The research leading to these results was funded by the follwing projects: ANPCyT PICT 2013-0558 and Ecos Sud MINCyT A16E03.

\section{References}

Abuzaid, W. Z., Sangid, M. D., Carroll, J. D., Sehitoglu, H. \& Lambros, J. (2012). J. Mech. Phys. Solids, 60, 1201-1220.

Allain-Bonasso, N., Wagner, F., Berbenni, S. \& Field, D. P. (2012). Mater. Sci. Eng. A, 548, 56-63.

Bachmann, F., Hielscher, R. \& Schaeben, H. (2011). Ultramicroscopy, 111, 1720-1733.

Bieler, T. R., Eisenlohr, P., Roters, F., Kumar, D., Mason, D. E., Crimp, M. A. \& Raabe, D. (2009). Int. J. Plast. 25, 1655-1683.

Bieler, T. R., Eisenlohr, P., Zhang, C., Phukan, H. J. \& Crimp, M. A. (2014). Curr. Opin. Solid State Mater. Sci. 18, 212-226.

Calcagnotto, M., Ponge, D., Demir, E. \& Raabe, D. (2010). Mater. Sci. Eng. A, 527, 2738-2746.

Franciosi, P. \& Berbenni, S. (2008). Int. J. Plast. 24, 1549-1580.

Githinji, D. N., Northover, S., Bouchard, P. J. \& Rist, M. (2013). Metall. Mater. Trans. A, 44, 4150-4167.

Gurao, N. P. \& Suwas, S. (2013). Mater. Lett. 99, 81-85.

Jorge-Badiola, D., Iza-Mendia, A. \& Gutiérrez, I. (2007). J. Microsc. 228, 373-383.

Kamaya, M. (2011). Ultramicroscopy, 111, 1189-1199.

Kamaya, M. (2012). Mater. Charact. 66, 56-67.

Kamaya, M., Wilkinson, A. J. \& Titchmarsh, J. M. (2005). Nucl. Eng. Des. 235, 713-725.

Kanjarla, A. K., Van Houtte, P. \& Delannay, L. (2010). Int. J. Plast. 26, 1220-1233.

Kashihara, K. \& Wert, J. A. (2006). Mater. Trans. 47, 233-238.

Lebensohn, R. A., Brenner, R., Castelnau, O. \& Rollett, A. D. (2008). Acta Mater. 56, 3914-3926.

Mishra, A., Mishra, H. \& Nayakankuppam, D. (2009). Psychological Sci. 20, 867-870.

Moussa, C., Bernacki, M., Besnard, R. \& Bozzolo, N. (2015). IOP Conf. Ser. Mater. Sci. Eng. 89, 012038.

Pouillier, E., Gourgues, A.-F., Tanguy, D. \& Busso, E. P. (2012). Int. J. Plast. 34, 139-153.

Rollett, A. D., Brahme, A. P. \& Roberts, C. G. (2007). Mater. Sci. Forum, 558-559, 33-42.

Rollett, A. D., Wagner, F., Allain-Bonasso, N., Field, D. \& Lebensohn, R. A. (2012). Mater. Sci. Forum, 702-703, 463-468.

Schayes, C., Bouquerel, J., Vogt, J. B., Palleschi, F. \& Zaefferer, S. (2016). Mater. Charact. 115, 61-70.

Scheriau, S. \& Pippan, R. (2008). Mater. Sci. Eng. A, 493, 48-52.

Schwartz, A. J., Kumar, M. \& Adams, B. L. (200). Electron Backscatter Diffraction in Materials Science, 2nd ed. New York: Kluwer Academic/Plenum Publishers.

Signorelli, J. W., Serenelli, M. J. \& Bertinetti, M. A. (2012). J. Mater. Process. Technol. 212, 1367-1376.

Soer, W. A., Aifantis, K. E. \& Dehosson, J. (2005). Acta Mater. 53, 4665-4676. 
Soifer, Y. M., Verdyan, A., Kazakevich, M. \& Rabkin, E. (2002). Scr. Mater. 47, 799-804.

Subedi, S., Pokharel, R. \& Rollett, A. D. (2015). Mater. Sci. Eng. A, 638, 348-356.

Takayama, Y., Szpunar, J. A. \& Kato, H. (2005). Mater. Sci. Forum, 495-497, 1049-1054.

Thorning, C., Somers, M. A. J. \& Wert, J. A. (2005). Mater. Sci. Eng. A, 397, 215-228.
Vachhani, S. J., Doherty, R. D. \& Kalidindi, S. R. (2016). Int. J. Plast. 81, 87-101.

Wagner, F., Allain-Bonasso, N., Berbenni, S. \& Field, D. P. (2012). Mater. Sci. Forum, 702-703, 245-252.

Winther, G. (2008). Mater. Sci. Eng. A, 483-484, 40-46.

Wright, S. I., Field, D. P. \& Nowell, M. M. (2015). IOP Conf. Ser. Mater. Sci. Eng. 89, 012049.

Yoda, R., Yokomaku, T. \& Tsuji, N. (2010). Mater. Charact. 61, 913-922. 